EPJ manuscript No.

(will be inserted by the editor)

\title{
Direct experimental text of non-separability and other quantum techniques using continuous variables of light
}

\author{
N. Korolkova, Ch. Silberhorn, O. Glöckl, S. Lorenz, Ch. Marquardt, G. Leuchs \\ Zentrum für Moderne Optik an der Universität Erlangen-Nürnberg, Staudtstraße 7/B2, D-91058 Erlangen, Germany.
}

Received: date / Revised version: date

\begin{abstract}
We present schemes for the generation and evaluation of continuous variable entanglement of bright optical beams and give a brief overview of a variety of optical techniques and quantum communication applications on this basis. A new entanglement-based quantum interferometry scheme with bright beams is suggested. The performance of the presented schemes is independent of the relative interference phase which is advantageous for quantum communication applications.
\end{abstract}

PACS. 03.67.Hk Quantum commmunication - 42.50.Dv Quantum optics; Nonclassical field states

\section{Introduction}

Quantum information processing using continuous variables [1,2,3] has emerged as an alternative approach to quantum information with single photons and atoms described with discrete variables. The main motivation for this new approach is the availability of controlled sources, efficient detection systems and easy-to-handle processing using linear elements. The price one has to pay for these advantages is always non-maximal entanglement. What is continuous variable entanglement about? We first give a short introduction providing an intuitive picture of how continuous variable entanglement manifests itself. Con- sider two spatially separated optical modes $j=1,2$. The involved optical fields can be fully described by means of field quadratures 顿, the amplitude quadrature $\hat{X}_{j}=$ $\hat{a}_{j}^{\dagger}+\hat{a}_{j}$ and the phase quadrature $\hat{Y}_{j}=i\left(\hat{a}_{j}^{\dagger}-\hat{a}_{j}\right)$. They obey the following commutation rules:

$$
\left[\hat{X}_{j}, \hat{Y}_{k}\right]=i \delta_{j k}, j, k=1,2 .
$$

These commutation relations leave the possibility for combined variables of both modes to commute:

$$
\left[\hat{X}_{1}+\hat{X}_{2}, \hat{Y}_{1}-\hat{Y}_{2}\right]=0
$$

Hence quantum states are possible, for which all the variables $\hat{X}_{j}, \hat{Y}_{j}$ are uncertain, but certain joint variables of 
two optical modes together are both well defined:

$$
\begin{array}{r}
\hat{X}_{1}+\hat{X}_{2} \rightarrow \text { well defined }, \\
\hat{Y}_{1}-\hat{Y}_{2} \rightarrow \text { well defined. }
\end{array}
$$

In the quantum optical context this is known as two-mode squeezing for $\hat{X}$ or $\hat{Y}$ 四. The measure for the degree of two-mode squeezing is the variances of the sum or difference signal of two modes, which approache zero for perfect squeezing:

$$
\begin{gathered}
V\left(\hat{X}_{1}+\hat{X}_{2}\right) \rightarrow 0, \\
V\left(\hat{Y}_{1}-\hat{Y}_{2}\right) \rightarrow 0,
\end{gathered}
$$

where $V(A)=\left\langle\hat{A}^{2}\right\rangle-\langle\hat{A}\rangle^{2}$ denotes a variance of an operator $\hat{A}$. Equations (4) also represent a measure for quantum correlations between two spatially separated optical modes, i.e. for continuous variable entanglement.

\section{Experimental evaluation of continuous variable entanglement}

Can we indeed associate such kind of correlations with entanglement? An entangled state is a non-separable quantum state. It means that the state of a system cannot be represented as a convex sum of product states of two subsystems. The necessary and sufficient condition for separability of a system for discrete variables is given by the Peres-Horodecki criterion [5]. This condition has been recently extended to continuous variable two mode Gaussian states [6,7], like two mode squeezed states (14). The criterion derived by Duan et al has an attractive potential for experimental quantum communication as it can be expressed in terms of observable quantities. They can then be measured using the conventional toolbox of experimental quantum optics. In this spirit we re-express the results of [6, [] in terms of the amplitude $\hat{X}_{j}=\hat{a}_{j}^{\dagger}+\hat{a}_{j}$ and the phase $\hat{Y}_{j}=i\left(\hat{a}_{j}^{\dagger}-\hat{a}_{j}\right)$ quadratures of bright beams $j=1,2$. Because of the high intensity of the optical fields involved, we use the linearization approach throughout the paper: $\hat{X}_{\mathrm{j}}=\left\langle X_{\mathrm{j}}\right\rangle+\delta \hat{X}_{\mathrm{j}}, \hat{Y}_{\mathrm{j}}=\left\langle Y_{\mathrm{j}}\right\rangle+\delta \hat{Y}_{\mathrm{j}}$. The entangled quantities are then the quantum uncertainties in the respective field quadratures. The non-separability criterion for the quantum state of two optical modes requires:

$$
\begin{gathered}
V_{\mathrm{sq}}^{ \pm}(\delta X)+V_{\mathrm{sq}}^{\mp}(\delta Y)<2, \\
V_{\mathrm{sq}}^{ \pm}(\delta X)=\frac{V\left(\delta \hat{X}_{1} \pm g \delta \hat{X}_{2}\right)}{V\left(\hat{X}_{1, \mathrm{SN}}+g \hat{X}_{2, \mathrm{SN}}\right)}, \\
V_{\mathrm{sq}}^{\mp}(\delta Y)=\frac{V\left(\delta \hat{Y}_{1} \mp g \delta \hat{Y}_{2}\right)}{V\left(\hat{Y}_{1, \mathrm{SN}}+g \hat{Y}_{2, \mathrm{SN}}\right)}
\end{gathered}
$$

where $g$ is a variable gain. The variances labeled "SN" correspond to the shot noise level of respective beams which marks a boundary between classical and quantum regime. The upper (lower) signs hold for the anti-correlated (correlated) amplitude quadratures and correlated (anti-correlated) phase quadratures. The squeezing variances in Eq. (5, 6, 7) are the normalized variances of Eq. (4). $V_{\mathrm{sq}}=1$ corresponds, e.g. to coherent states in both optical modes and $V_{\mathrm{sq}}<1$ corresponds to two mode squeezing, hence the name squeezing variances. They are quantities measurable in an experiment and will be used throughout this paper for the experimental evaluation of continuous variable entanglement. 


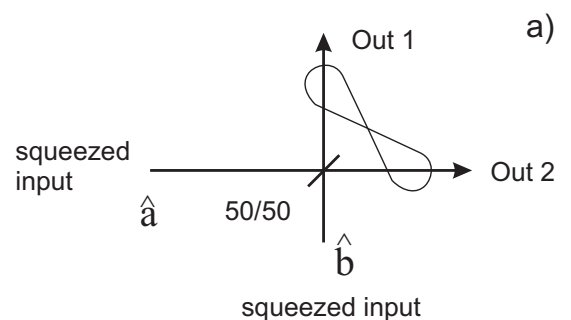

b)

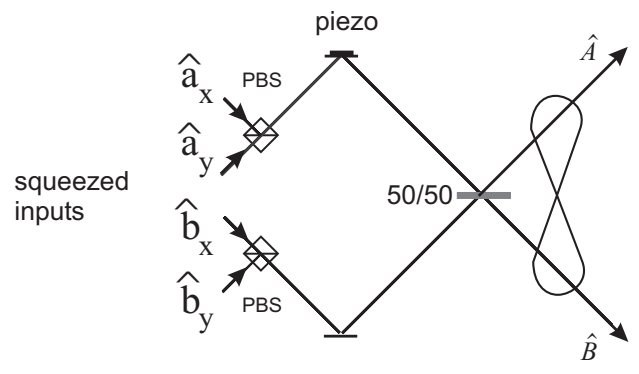

Fig. 1. Schemes for generation of continuous variable entanglement by linear interference of squeezed beams: a) Entanglement of amplitude and phase quadratures; b) Continuous variable polarization entanglement. Operators $\hat{a}, \hat{b}$ describe input bright squeezed beams, indices $x, y$ denote two orthogonal polarization modes, 50/50 is a beam splitter with a respective splitting ratio and PBS stands for a polarization beam splitter.

\subsection{Entanglement of amplitude and phase quadratures}

The generation of entanglement with continuous variables typically uses optical parametric down conversion in a subthreshold OPO. This process creates two vacuum states with quantum correlated amplitude and anti-correlated phase quadratures or vice versa [8,9,10]. Another promising scheme for the generation of EPR-entangled beams utilizes the superposition of two independently squeezed bright light fields to create quantum correlations (11, 12. and Figure 1 (a)). For this purpose the optical phases of the incoming fields are chosen such that the initial fields acquire a phase difference of $\frac{\pi}{2}$ for the linear interference at a beam splitter. This scheme was recently implemented using the Kerr-nonlinearity of an optical fibre to produce two bright amplitude squeezed pulsed light fields [13]. Interference of these two beams generates EPR-entanglement with anti-correlated amplitude quadrature and correlated phase quadrature with $V_{\text {sq }}^{ \pm}(\delta X)+V_{\text {sq }}^{\mp}(\delta Y)=0.80 \pm 0.06$ [13.

\subsection{Polarization entanglement}

Entanglement of the amplitude and phase quadratures is familiar but of course not the only possible type of continuous variable entanglement. Another promising set of continuous variables are the polarization variables, the quantum Stokes operators (see [14] and references therein).

The Stokes operators are defined in analogy to the Stokes parameters in classical optics:

$$
\begin{array}{r}
\hat{S}_{0}=\hat{n}_{x}+\hat{n}_{y}, \\
\hat{S}_{1}=\hat{n}_{x}-\hat{n}_{y}, \\
\hat{S}_{2}=\hat{a}_{x}^{\dagger} \hat{a}_{y}+\hat{a}_{y}^{\dagger} \hat{a}_{x}, \\
\hat{S}_{3}=i\left[\hat{a}_{y}^{\dagger} \hat{a}_{x}-\hat{a}_{x}^{\dagger} \hat{a}_{y}\right]
\end{array}
$$

where $\hat{a}_{k}^{\dagger}\left(\hat{a}_{k}\right)$ are photon creation (annihilation) operators and $\hat{n}_{k}=\hat{a}_{k}^{\dagger} \hat{a}_{k}$ is the photon number operator in polarization mode $k$ with the basic set of the orthogonal modes linearly polarized along $x$ and $y$ directions $(k=x, y)$. The operators $\hat{a}_{k}, \hat{a}_{l}^{\dagger}$ obey the usual commutation relation $\left[\hat{a}_{k}, \hat{a}_{l}^{\dagger}\right]=\delta_{k l},(k, l=x, y)$. The operators $\hat{S}_{j}(\mathrm{j}=1,2,3)$ satisfy the commutation relations of the Lie algebra of the 
$\mathrm{SU}(2)$ group, for example:

$$
\left[\hat{S}_{2}, \hat{S}_{3}\right]=i 2 \hat{S}_{1}
$$

The other relations are obtained from (12) by cyclic permutation of the labels.

There are quantum uncertainties associated with each of the Stokes operators (8611). A quantum state of an optical field is said to be polarization squeezed if the uncertainty in one of the Stokes operators is reduced below that of coherent light, i.e. $V_{j} \leq V^{c o h}(j=1,2,3)$, at the cost of increased uncertainty in the other Stokes parameters 14. Such nonclassical polarization squeezed states can be generated by superimposing two orthogonally polarized amplitude squeezed light beams on a polarizing beam splitter [14].

Polarization entanglement of continuous variables can in turn be generated by linear interference of two polarization squeezed beams. This is in analogy to the interference of amplitude squeezed beams 12,13 for the generation of bright EPR entanglement of the amplitude and phase quadratures (Fig. 1).

For bright beams, polarization entanglement implies quantum correlations of the uncertainties of the Stokes operators in two spatially separated optical modes $\hat{A}, \hat{B}$ (Fig. 1 (b)). Let us assume that polarization squeezed beams for entanglement generation are produced by interference of orthogonally polarized amplitude squeezed beams with equal squeezing $V(\delta X)<1$ in each of the four input modes (Fig. 1 (b)). It follows from (811, 12) that for input beams of equal amplitude only operators $\hat{S}_{1 A, B}$ and $\hat{S}_{3 A, B}$ are conjugate variables because only
$V\left(S_{3}\right) V\left(S_{1}\right) \geq\left|\left\langle\hat{S}_{2}\right\rangle\right| \neq 0$. The non-separability of the quantum polarization state of two modes $\hat{A}$ and $\hat{B}$ then means [14]:

$$
V_{\mathrm{sq}}^{+}\left(\delta S_{1}\right)+V_{\mathrm{sq}}^{-}\left(\delta S_{3}\right)<2
$$

with the squeezing variances equal to:

$$
\begin{gathered}
V_{\mathrm{sq}}^{+}\left(\delta S_{1}\right)=\frac{V\left(\delta \hat{S}_{1 A}+g \delta \hat{S}_{1 B}\right)}{V\left(\hat{S}_{1 A, \mathrm{SN}}+g \hat{S}_{1 B, \mathrm{SN}}\right)}=V(\delta X), \\
V_{\mathrm{sq}}^{-}\left(\delta S_{3}\right)=\frac{V\left(\delta \hat{S}_{3 A}-g \delta \hat{S}_{3 B}\right)}{V\left(\hat{S}_{3 A, \mathrm{SN}}+g \hat{S}_{3 B, \mathrm{SN}}\right)}=V(\delta X) .
\end{gathered}
$$

The important advantage of such non-classical polarization states is that it is possibile to measure the relevant conjugate variables by direct detection only, using linear optical elements.

\section{Optical techniques in direct detection}

The use of bright beams for entanglement generation allows the implementation of a range of optical techniques with a particularly simple detection system. Unlike many schemes described elsewhere, those proposed here do not require explicit measurements of the phase quadrature of the light field. This advantage is brought about by the use of a simple interferometric setup which avoids more cumbersome measurements, in particular those involving local oscillator fields. In the following sections we describe these techniques in detail using the entanglement of field quadratures as an example (Sec. 2.1 and Fig. 1 (a)).

Prior to doing this it is worthwhile to contrast quadrature entanglement and polarization entanglement. For polarization entanglement (Sec. 2.2 and Fig. 1 (b)) the explicit measurement of all the relevant conjugate variables 
and of their variances can be performed in direct detection [14]. For example, it is straightforward to record $V_{\mathrm{sq}}^{+}\left(\delta S_{1}\right)$, $V_{\text {sq }}^{-}\left(\delta S_{3}\right)$ and calculate 13 . It is thus not necessary to detect the non-separability of polarization state in an indirect way. If the schemes illustrated in the following sections were though to use polarization entanglement, they would be more sophisticated, requiring additional linear elements, such as half-wavelength plates and polarizers, to record the variances of the Stokes operators 14.

Thus both quadrature and polarization entanglement have their advantages and disadvantages. These determine the scope of applications of the two different entanglement types as discussed in Sec. 2.1 and 2.2. Continuous variable polarization entanglement is best suited to schemes requiring explicit measurements of the involved conjugate variables, e.g. to quantum cryptography. In contrast, quadrature entanglement is more appropriate for schemes involving direct intensity detection, e.g. quantum dense coding, quantum teleportation.

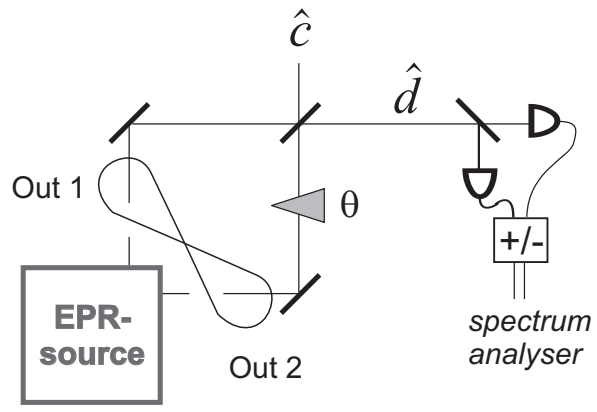

Fig. 2. Direct detection of the non-separability criterion

\subsection{Measuring non-separability of a quantum state}

In this section we show how the non-separability of the quantum states described in Sec. 2.1, 2.2 can be verified in an experiment. To determine the non-separability criterion of Eq. (5), the EPR source is treated as a black box. We would like to test now for continuous variable entanglement of the two output optical modes of Sec. 2.1 and Figure 1 (a). These two modes are superimposed at a beam splitter (Fig. 2). The field operators of the beams after this interference are denoted $\hat{c}, \hat{d}$. To adjust the relative interference phase, a variable phase shift $\theta$ can be introduced in one of the arms. The balanced detection is performed in one of the output arms, $\hat{c}$ or, equivalently, $\hat{d}$. Difference and sum photocurrents are recorded and the relative phase $\theta$ is scanned. The difference photocurrent provides the shot noise level reference:

$$
V\left(\delta X_{\mathrm{c}, \mathrm{d}, \mathrm{SN}}\right)=\alpha^{2}(1 \pm \cos \theta)
$$

The two signs refer to the two complementary outputs. The sum photocurrent delivers the amplitude noise variance of the signal in the output $\hat{c}$ or $\hat{d}$ :

$$
\begin{array}{r}
\left\langle\left(\delta X_{\mathrm{c}, \mathrm{d}}\right)^{2}\right\rangle= \\
\frac{1}{2} \alpha^{2}\left[(1 \pm \cos \theta)^{2} V_{\mathrm{sq}}^{+}(\delta X)+\sin ^{2} \theta V_{\mathrm{sq}}^{-}(\delta Y)\right]
\end{array}
$$

From the measurements 16,17 the normalized noise variance can be inferred:

$$
\begin{array}{r}
V\left(\delta X_{\mathrm{c}, \mathrm{d}}\right)=\frac{\left\langle\left(\delta X_{\mathrm{c}, \mathrm{d}}\right)^{2}\right\rangle}{V\left(\delta X_{\mathrm{c}, \mathrm{d}, \mathrm{SN}}\right)}= \\
\frac{1}{2}\left[(1 \pm \cos \theta) V_{\mathrm{sq}}^{+}(\delta X)+\frac{\sin ^{2} \theta}{(1 \pm \cos \theta)} V_{\mathrm{sq}}^{-}(\delta Y)\right] .
\end{array}
$$

It contains the information about both relevant squeezing variances $V_{\mathrm{sq}}^{+}(\delta X), V_{\mathrm{sq}}^{-}(\delta Y)$ which characterize the quan- 
tum correlations between optical modes 1,2 (Fig. 2). For $\theta=\frac{\pi}{2}$ the measured noise variance 18 ) is directly proportional to the Peres-Horodecki criterion (5) for continuous variables:

$$
V\left(\delta X_{\mathrm{c}, \mathrm{d}}\right)=\frac{1}{2}\left[V_{\mathrm{sq}}^{+}(\delta X)+V_{\mathrm{sq}}^{-}(\delta Y)\right]
$$

If the interfering amplitude squeezed beams are of equal squeezing, the output entangled beams have symmetric circular uncertainty regions and $V_{\mathrm{sq}}^{+}(\delta X)=V_{\mathrm{sq}}^{-}(\delta Y)=$ $V_{\mathrm{sq}}$. Then equation (18) takes the form:

$$
V\left(\delta X_{\mathrm{c}, \mathrm{d}}\right)=\frac{\left\langle\left(\delta X_{\mathrm{c}, \mathrm{d}}\right)^{2}\right\rangle}{V\left(\delta X_{\mathrm{c}, \mathrm{d}, \mathrm{SN}}\right)}=V_{\mathrm{sq}}
$$

and the non-separability condition reads: $V_{\mathrm{sq}}<1$ independent of the relative interference phase $\theta$. Using (19) or (20), the non-separability of the state can be reliably verified experimentally using the interferometric scheme of Fig. 3.

\subsection{Quantum interferometry}

The interferometric detection of the non-separability criterion provides a new and interesting insight into the performance of the scheme (Fig. 2) as a setup for high-precision measurements of small phase modulations. There are quantum limitations to the sensitivity of optical interferometers in addition to mechanical, thermal, and other effects. For example, the standard quantum limit for minimal resolvable phase modulations derived from the Heisenberg uncertainty principle reads:

$$
\delta \theta_{\min }=\sqrt{\frac{1}{n}}
$$

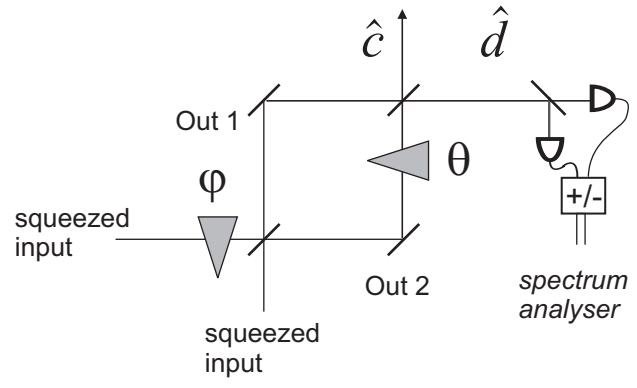

Fig. 3. Set up for quantum interferometry

where $n$ is a total photon number in the measured mode. Quantum interferometry attempts to exploit non-classical states of light to go beyond this standard quantum limit in high-sensitive measurements $[4,15]$.

Consider the operation of the setup depicted in Figure 3 in more detail [16]. Compared to the setups in Figures 1 and 2, the scheme of Fig. 通 in addition allows the phase $\varphi$ of the first interference to be controlled. That means that the degree of entanglement between the output beams 1 and 2 is tunable. The noise variances and mean value of the signal at the output of the interferometer are recorded by balanced detection in at least one of the output ports (see also Fig. 2). From these measurements the noise variance of the signal normalized to its shot noise level can be inferred: $V\left(\delta X_{c, d}\right)=\left\langle\left(\delta X_{\mathrm{c}, \mathrm{d}}\right)^{2}\right\rangle / V\left(\delta X_{\mathrm{c}, \mathrm{d}, \mathrm{SN}}\right)$. In this way the noise reduction at the output of the interferometer can be evaluated with respect to the interference phases $\varphi$ and $\theta$. This is illustrated in Figure 4 .

For the phase of the first interference equal to zero, $\varphi=0$, no entanglement is generated. In this case the setup (Fig. 3) represents just a trivial Mach-Zehnder interferometer with two squeezed inputs transformed to two squeezed outputs $\hat{c}, \hat{d}$, if the phase of the second interfer- 


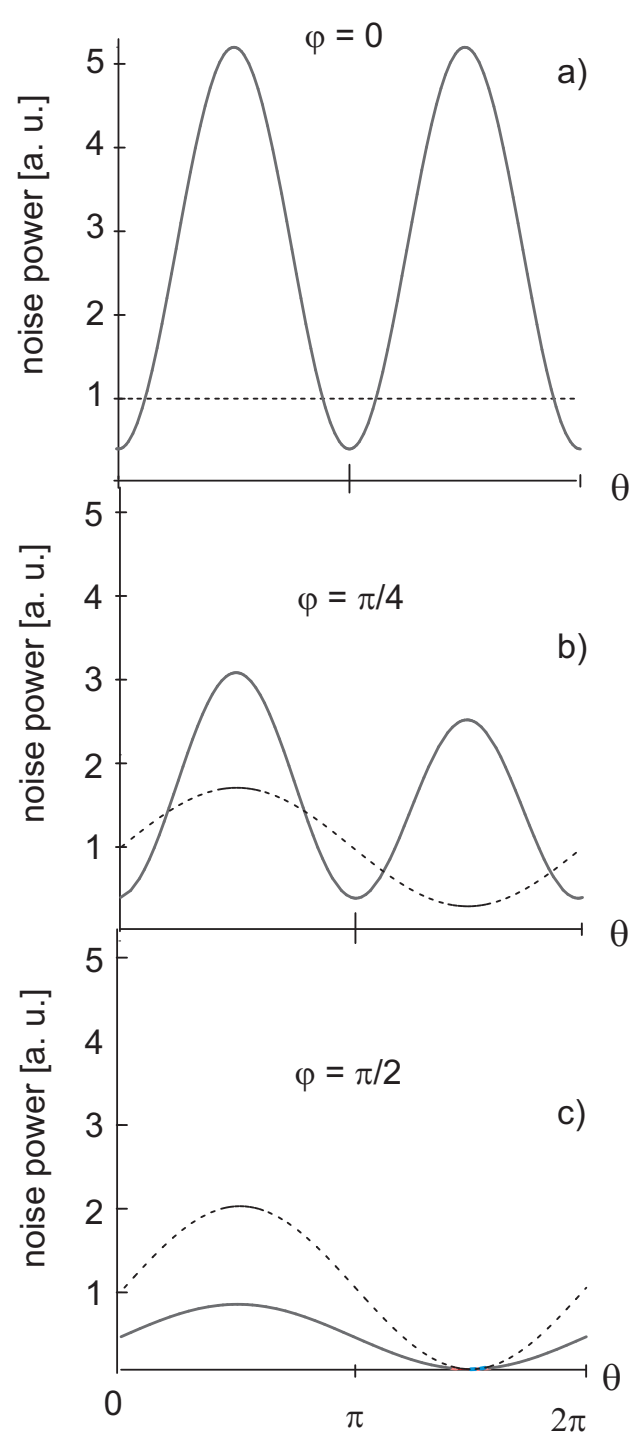

Fig. 4. Intensity noise variances (solid line) and corresponding shot noise levels (dashed) at the output of the interferometer in Figure 3 versus variable phase shift $\theta$ for different values of $\varphi$. The curves are plotted for $4 \mathrm{~dB}$ input squeezing (see Ref. [13]).

ence is adjusted properly (Fig. $1(\mathrm{a})$ ). The phase relation equal to $\varphi=\frac{\pi}{2}$ corresponds to the maximal available entanglement between beams 1 and 2 (Fig. A (c)). This is the situation described in the previous section (Fig. 2). The dependence of the second interference on the phase is given then by equation $(20)$. Note that noise reduction observed for $\varphi=\frac{\pi}{2}$ is insensitive to the phase $\theta$ : the ratio $V\left(\delta X_{c, d}\right)=\left\langle\left(\delta X_{\mathrm{c}, \mathrm{d}}\right)^{2}\right\rangle / V\left(\delta X_{\mathrm{c}, \mathrm{d}, \mathrm{SN}}\right)<1$ remains constant and less than unity for all values of $\theta$ (Fig. 4 (c)). Figure 4 (b) shows the intermediate situation with a close to maximal degree of entanglement (see also [13).

What do all these noise variances have to do with quantum interferometry? The proper analysis of the curves gives an important hint: the main mechanism enabling the suppression of the resolution limit below that of Eq. (21) is the quantum correlation, i.e. entanglement, between beams 1 and 2 . To make this statement comprehensive, let us discuss Figure 4 again in view of quantum interferometry.

Recall that the limit for minimal resolvable phase modulation can be derived in terms of signal-to-noise ratio [4]:

$$
\mathrm{SNR}=\frac{\langle\hat{n}\rangle}{\sqrt{V(n)}}=\frac{\left\langle\hat{X}_{c, d}\right\rangle}{\sqrt{V\left(\delta X_{c, d}\right)}}
$$

where $\hat{n}=\hat{c}^{\dagger} \hat{c}$ is the photon number operator in mode $\hat{c}$ with the mean value $\langle\hat{n}\rangle=n$ (analogously for $\hat{d}$ ). The smallest detectable phase shift is defined to be that for which $\mathrm{SNR}=1$.

The analysis of Figure 4 on this basis yields the following results. For the phase relation $\varphi=0$ (Fig. 由 (a)) there is no interferometric performance of the setup as the signal is zero. For Figure 4 (b) minimal resolvable phase modulations around $\theta=0+k \pi$ are:

$$
\delta \theta_{\min }=\sqrt{\frac{2 V_{\mathrm{sq}}^{+}(\delta X)}{n}}
$$

where $V_{\mathrm{sq}}^{+}(\delta X)=V(\delta X)$ is the squeezing variance determining the maximal available quality of entanglement for 


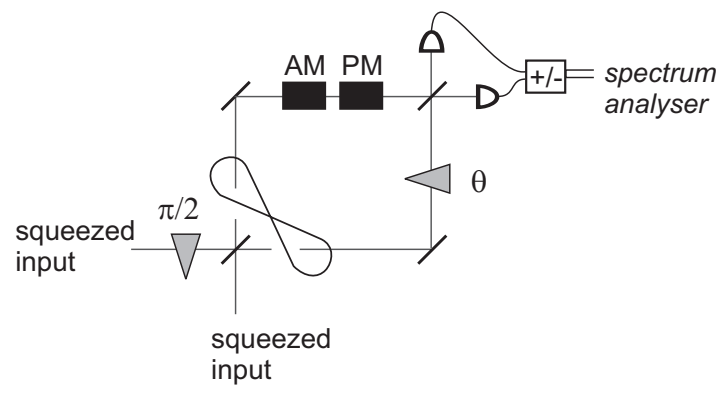

Fig. 5. Setup for quantum dense coding with bright entangled beams.

the input squeezed beams with $V(\delta X)$. For the situation corresponding to maximal available entanglement between the beams (Fig. 目(c)) the minimal resolvable phase modulations around $\theta=\frac{\pi}{2}+k \pi$ drop out as:

$$
\delta \theta_{\min }=\sqrt{\frac{V_{\mathrm{sq}}^{+}(\delta X)}{n}} .
$$

The setup thus reaches the best performance for the best entanglement.

First experiments on quantum interferometry were performed using squeezed vacuum at the second normally not used input of a Mach-Zehnder or a Michelson interferometer 18, 19]. The important requirement for reaching sub-shot noise operation is that the squeezed quadrature of the second input port is $\frac{\pi}{2}$ out of phase with the coherent excitation of the first input port. If one has two intense beams at each input port this condition can still be fulfilled [17. The scheme considered in Fig. 3 is closely related to quantum interferometry with two Fock states [20] and to other interferometric setups used before 4,17 , 20], i.e. it uses two $\varphi=\pi / 2$ shifted bright squeezed beams as input modes. However, it possess several novel features. First, noise reduction performance of the setup is insen- sitive to the phase $\theta$ for symmetric beams (Fig. 1 (c)). Second, so far the ability to suppress the quantum limit of the interferometer was always related to reduced noise in the input fields. Here it is clearly demonstrated that it is the quantum correlations, quantum entanglement, between the interferometer arms which is responsible for the enhanced resolution (see also [21]). Third, it is not necessary to go for the detection of the difference signal of two outputs $\hat{c}^{\dagger} \hat{c}-\hat{d}^{\dagger} \hat{d}$ because the measurement in one arm provides the same precision. Fourth, as the noise reduction is phase insensitive, one can choose an arbitrary point of operation $\theta \neq \pi / 2,0$ which might be advantageous in a particular application.

\subsection{Quantum dense coding}

The phase-insensitive noise reduction performance of the interferometric scheme (Fig. 3) makes it a promising setup for dense coding [22,23]. Quantum dense coding was first suggested [22] and realized in an experiment [25] for discrete quantum variables like polarization states of entangled photon pairs. In this case it aims to enhance the classical information capacity of quantum communication channels beyond the Holevo's bound 24. Recently the idea of quantum dense coding was extended to the quadrature components of the electromagnetic field using entangled light beams as quantum channel [23]. For such continuous variables, dense coding refers to the ability to read out amplitude and phase modulations with a precision below the limit given by the Heisenberg uncertainty relation. For large photon numbers, the channel capac- 
ity of the scheme [23] approaches twice that of classical coherent-state communication [23]. The first experimental implementation of quantum dense coding with bright entangled beams was proposed in Ref. [26] and recently implemented 27.

Figure 5 shows the interferometric setup (Fig. 3) modified to implement dense coding using intense entangled beams. This corresponds to the setup shown in Ref. [26] where this scheme was only briefly addressed. The analysis was based on the squeezing characteristics of the input beams for entanglement generation. Here we would like to emphasize the role of the quantum correlations $V_{\mathrm{sq}}^{+}(\delta X)$, $V_{\mathrm{sq}}^{-}(\delta Y)$ on the scheme performance, which means on being able to read out amplitude and phase modulations with a precision below the Heisenberg limit. We assume $V_{\mathrm{sq}}^{+}(\delta X)=V_{\mathrm{sq}}^{-}(\delta Y)=V_{\mathrm{sq}}$ which is required for the phase independent performance of the scheme. The balanced detection of the combined mode of both outputs delivers the information about small imposed amplitude $\delta X_{\mathrm{m}}$ and phase $\delta Y_{\mathrm{m}}$ modulations:

$$
\begin{gathered}
V^{+}=\alpha^{2}\left(V_{\mathrm{sq}}+V\left(\delta X_{\mathrm{m}}\right)\right), \\
V^{-}=\alpha^{2}\left(V_{\mathrm{sq}}+V\left(\delta Y_{\mathrm{m}}\right)\right)
\end{gathered}
$$

where $V^{ \pm}$are the noise variances recorded in the sum and difference detector channels, respectively. The Heisenberg relation imposes a lower resolution bound for coherent communication and there will be $V_{\text {coh }}=1$ in the place of $V_{\mathrm{sq}}$ in equations (25, 26). Quantum EPR-like correlations between the bright beams in the two interferometer arms imply $V_{\text {sq }} \ll 1$. Hence the use of quantum entangle- ment enhances the resolution of quadrature modulations beyond the Heisenberg limit for all values of $\theta$ (25, 26). This enables quantum dense coding independent of the interference phase $\theta$. The phase-insensitivity eliminates the need for extensive setup stabilization. It makes the scheme more reliable and easier to handle, in particular for the communication over long distances.

\section{Conclusions}

The generation and experimental characterization of bright beam entanglement has been reviewed. We report on an entanglement-based interferometric setup using intense optical fields (Fig. 2, 3, 5). This setup can be modified to implement various optical techniques ranging from entanglement evaluation through quantum interferometry and dense coding to quantum teleportation [2, 12]. In all these applications only direct detection is required without the need for local oscillator fields. The role of quantum EPR-like correlations, $V_{\mathrm{sq}}$, was explicitly shown, as well as

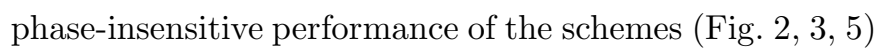
provided that there is equal squeezing in the input beams.

A phase-insensitive scheme for quantum interferometry with bright entangled beams is proposed (Fig. 3). The resolution limit for phase modulations can be suppressed up to $\sqrt{\frac{V_{\mathrm{sq}}(\delta X)}{n}}$ as compared to $\sqrt{\frac{1}{n}}$ where $V_{s q}(\delta X)$ is determined by the quantum amplitude correlations of the entangled beams.

In the schemes outlined above it was sufficient to infer the information about the phase quadrature from the noise variances obtained in direct amplitude detection. How- 
ever, there are a number of quantum information protocols which require the explicit measurement of all involved conjugate quantum variables, for example, quantum key distribution with continuous variables (28, 29] and references therein). Continuous variable polarization entanglement 14 can be extremely useful for such purposes. For this type of entanglement all the relevant variables can be detected without the need for auxillary local oscillator fields or other evolved measurement techniques.

This work was supported by the Deutsche Forschungsgemeinschaft and by the EU grant under QIPC, project IST-1999-13071 (QUICOV). The authors gratefully acknowledge the help of J. Heersink in the preparation of the manuscript.

\section{References}

1. S. Lloyd and S. L. Braunstein, Phys. Rev. Lett. 82, (1999) 1784.

2. S. L. Braunstein and H. J. Kimble, Phys. Rev. Lett. 80, (1998) 869.

3. M. D. Reid and P. D. Drummond, Phys. Rev. Lett. 60, (1988) 2731; M. D. Reid, Phys. Rev. A 40, (1989) 913.

4. D. F. Walls and G. J. Milburn, Quantum Optics (Springer Verlag, Berlin Heidelberg 1994).

5. A. Peres, Phys. Rev. Lett. 77, (1996) 1413; M. Horodecki, P. Horodecki, and R. Horodecki, Phys. Lett. A 223, (1996) 1; P. Horodecki, Phys. Lett. A 232, (1997) 333.

6. L. -M. Duan, G. Giedke, J. I. Cirac, and P. Zoller, Phys. Rev. Lett. 84, (2000) 2722.

7. R. Simon, Phys. Rev. Lett. 84, (2000) 2726.
8. Z. Y. Ou, S. F. Pereira, H. J. Kimble, and K. C. Peng, Phys. Rev. Lett. 68, (1992) 3663.

9. A. Furusawa, J. L. Sørensen, S. L. Braunstein, C. A. Fuchs, H. J. Kimble, and E. S. Polzik, Nature 283, (1998) 706.

10. Y. Zhang, H. Wang, X. Li, J. Jing, C. Xie, and K. Peng, Phys. Rev. A 62, (2000) 023813.

11. U. Leonhardt, Measuring the quantum state of light (Cambridge University Press 1997).

12. G. Leuchs, T. C. Ralph, Ch. Silberhorn, and N. Korolkova, J. Mod. Opt. 46, (1999) 1927.

13. Ch. Silberhorn, P. K. Lam, O. Weiß, F. König, N. Korolkova, and G. Leuchs, Phys. Rev. Lett. 86, (2001) 4267.

14. N. Korolkova, G. Leuchs, R. Loudon, T.C. Ralph, and Ch. Silberhorn, Polarization Squeezing and Continuous Variable Polarization Entanglement, Phys. Rev. A, (2001) submitted.

15. C. M. Caves, Phys. Rev. D 23, (1981) 1693.

16. Ch. Silberhorn and N. Korolkova, Entanglement, quantum interferometry and dense coding with intense light pulses. Invited talk at Conference on Laser and ElectroOptics / Europe - European Quantum Electornics Conference CLEO/Europe-EQEC Focus Meeting 2001, Munich, Germany, June 18-22, 2001, CLEO/Europe-EQEC Focus Meeting Conference Digest, 309.

17. S. Inoue, Y. Yamaoto, and G. Bjork, Sub-shot-noise interferometer driven by amplitude squeezed light from an injection-locked semicnductor laser, International Quantum Electronics Conference IQEC 1996, Sydney, Australia, July 14-19, 1996, IQEC'1996 Technical Digest, ThH3, 210.

18. M. Xiao, L.-A. Wu, and H.J. Kimble, Phys Rev. Lett. 59, (1987) 278 .

19. S. Inoue, Y. Yamamoto, Phys. Lett. A 236, (1997) 183. 
20. M.J. Holland and K. Burnett, Phys. Rev. Lett. 71, (1993) 1355.

21. T. Kim, O. Pfister, M.J. Holland, J. Noh, and J. L. Hall, Phys. Rev. A 57, (1998) 4004.

22. C. H. Bennett and S. J. Wiesner, Phys. Rev. Lett. 69, (1992) 2881.

23. S. L. Braunstein and H. J. Kimble, Phys. Rev. A 61, (2000) 042302.

24. A. S. Holevo, IEEE Trans. Inf. Theory 44, (1998) 269.

25. K. Mattle, H. Weinfurter, P. G. Kwiat, and A. Zeilinger, Phys. Rev. Lett. 76, (1996) 4656.

26. J. Zhang and K. Peng, Phys. Rev. A 62, (2000) 064302.

27. J. Zhang, C. Xie, and K. Peng, Quantum switching and quantum secret sharing manipulated by squeezed state of light, Quantum Electronics and Laser Science Conference QELS 2001, Baltimore, Maryland, May 6-11, 2001, Advance Program, QThA3, 143.

28. S. Lorenz, Ch. Silberhorn, N. Korolkova, R.S. Windeler and G. Leuchs, Squeezed Light from Microstructured Fibres: towards Free Space Quantum Cryptography, Appl. Phys. B, (2001) in press.

29. Ch. Silberhorn, N. Korolkova, and G. Leuchs, Quantum cryptography with bright entangled beams, International Quantum Electronics Conference IQEC 2000, Nice, France, September 10-15, 2000, IQEC'2000 Conference Digest, QMB6, 8; E-print ArXiv: quant-ph/0109009 (2001). 